\title{
Importancia de la anamnesis exhaustiva ante una ginecomastia unilateral inexplicada en un niño
}

\author{
Importance of the exhaustive anamnesis to an unexplained unilateral \\ gynecomastia in a child
}

\author{
Lic. Cristina Pérez-García ${ }^{a}$ Dra. Ana B. Ariza Jiméneza , Lic. Laura Camacho Lozano y \\ Lic. Carmen de la Cámara Moraño ${ }^{a}$
}

\section{RESUMEN}

La ginecomastia es el crecimiento de la mama por un desequilibrio hormonal entre estrógenos y andrógenos. Un crecimiento importante y unilateral requiere descartar patologías subyacentes. Una causa poco frecuente es la traumática, que provoca aumento de tamaño por estimulación repetida.

Se presenta el caso de un niño de 6 años con ginecomastia unilateral. Se destaca como único hallazgo en las pruebas complementarias hiperprolactinemia. Rehistoriando, se detecta una continua autoestimulación mamaria manual y oral a través de mordiscos de meses de evolución. Tras el cese del estímulo, se observa la involución de la mama y la normalización de los niveles de prolactina séricos.

Palabras clave: ginecomastia, prepuberal.

\begin{abstract}
Gynecomastia consists of breast enlargement due to a hormonal imbalance between estrogens and androgens. Unilateral and important breast growth requires ruling underlying pathologic disorders out. Mechanical cause is uncommon, causing enlargement by repeated stimulation.

We report a 6-year-old boy with unilateral gynecomastia. Hyperprolactinemia is the only abnormal finding at laboratory tests. After repeated inquiries, a continuous breast selfstimulation is detected. Its relation with gynecomastia is verified because prolactin normalizes and breast regressed in further revisions, after stopping stimulus.

Key words: gynecomastia, prepubertal.
\end{abstract}

http: / / dx.doi.org/10.5546/ aap.2018.e655

Cómo citar: Pérez-García C, Ariza Jiménez AB, Camacho Lozano L, de la Cámara Moraño C. Importancia de la anamnesis exhaustiva ante una ginecomastia unilateral inexplicada en un niño. Arch Argent Pediatr 2018;116(5):e655-e658.

a. Unidad de Endocrinología Pediátrica, Hospital Universitario Reina Sofía (Córdoba), España.

Correspondencia:

Lic. Cristina Pérez García: cris_pg91@hotmail.com

Financiamiento: Ninguno.

Conflicto de intereses: Ninguno que declarar.

Recibido: 25-10-2017

Aceptado: 28-5-2018

\section{INTRODUCCIÓN}

La ginecomastia consiste en el crecimiento de la glándula mamaria en el varón y es un proceso relativamente frecuente. ${ }^{1}$ Su presentación habitual es en el período neonatal y puberal. ${ }^{1}$ Puede presentarse de forma bilateral o unilateral y asociarse o no a dolor, tensión local, rubor o secreción. ${ }^{2}$ Es importante diferenciarla de la lipomastia, muy frecuente en la actualidad, debido a la gran prevalencia de obesidad infantil. ${ }^{3}$ La mayoría de estos casos son entidades benignas y autolimitadas, si bien un crecimiento importante y unilateral requiere el estudio endocrinológico e incluso oncológico para descartar causas patológicas subyacentes. Por el contrario, la ginecomastia de aparición prepuberal, especialmente, la unilateral, es rara, con pocos casos descritos en la literatura.

Las causas y mecanismos que influyen en la etiopatogenia de la ginecomastia en la edad pediátrica son las siguientes: ${ }^{4}$ causa idiopática $(25 \%)$, puberal $(25 \%)$, exposición sistémica o farmacológica a estrógenos (20\%) (Tabla 1), ${ }^{5}$ cirrosis/malnutrición (8\%), hipogonadismo primario $(8 \%)$, tumoral $(3 \%)$, hipogonadismo secundario $(2 \%)$, alteraciones tiroideas $(1,5 \%)$, insuficiencia renal $(1 \%)$ o enfermedades locales, como mastitis. ${ }^{5,6}$ Se presenta el caso de un niño con una ginecomastia de causa traumática, infrecuente en la clínica. ${ }^{7}$

\section{CASO CLÍNICO}

Niño de 6 años que acudió a la consulta de Endocrinología Pediátrica por el aumento de tamaño de la mama izquierda, de 3 meses de evolución. Negaron traumatismo previo y uso de sustancias o fármacos inductores, en forma oral o tópica, en la familia o en el niño. En la exploración, presentó buen estado general, con peso de 25,3 kg (p59, 0,23 desvíos estándar-DE- ) y talla de 122,5 cm (p59, 0,2 DE), con índice de masa corporal (IMC) de 16,86\% (p57, 0,2 DE), con genitales masculinos normales, estadio G1P1 de 
Tanner, botón mamario izquierdo de unos $3 \mathrm{~cm}$ de diámetro, sin hallazgos patológicos en la piel circundante ni galactorrea, sangrado, retracción, hiperemia, dolor ni adenopatías palpables en ningún nivel. La exploración de la mama derecha fue normal, sin hallazgos en el resto de la exploración. Indagando en la anamnesis, la madre refirió la autoestimulación manual y mordiscos continuos a modo de estereotipia del área afectada desde hacía tiempo. Se catalogó como ginecomastia de posible causa mecánica; se solicitó el estudio analítico y se citó para la revisión y la recogida de resultados en un mes. En esta consulta, aportó la radiografía de silla turca solicitada por otro especialista, sin hallazgos patológicos, y refirió que había cesado la estimulación de la mama, por lo que había disminuido su tamaño. En la analítica solicitada (Tabla 2), se destacó la elevación de niveles de $17-\mathrm{OH}$ progesterona (valores que se normalizaron en sucesivos controles analíticos) y prolactina de $25,52 \mu \mathrm{g} / \mathrm{L}$ obtenida mediante el ensayo por quimioluminiscencia, con hemograma, gonadotropinas, hormonas sexuales, andrógenos, fracción beta de la gonadotropina coriónica humana ( $\beta$-hCG), función hepática, renal y tiroidea normales. Se volvió a citar en 3 meses para control y se comprobó la desaparición de la ginecomastia y la normalización de los niveles de prolactina tras el cese del estímulo repetido, lo que confirmó la sospecha diagnóstica.

\section{DISCUSIÓN}

Se presenta el caso de un varón de 6 años, prepúber, con ginecomastia unilateral de causa mecánica, lo que supone una edad de presentación y etiología muy infrecuentes para este proceso.

La ginecomastia, aunque es, generalmente, un

TABla 1. Principales fármacos y sustancias inductoras de ginecomastia

\section{Hormonales \\ Psicofármacos \\ Fármacos cardiovasculares \\ Quimioterápicos \\ Antiulcerosos \\ Antibióticos \\ Drogas de abuso \\ Miscelánea}

\author{
Estrógenos, esteroides anabólicos, testosterona, HCG, antiandrógenos, GH \\ Fenotiazinas, antidepresivos tricíclicos, haloperidol, diazepam \\ Digoxina, calcioantagonistas, IECA, metildopa, reserpina, minoxidil \\ Agentes alquilantes \\ Omeprazol, ranitidina \\ Isoniacida, fluconazol, metronidazol, ketoconazol, antirretrovirales \\ Marihuana, alcohol, heroína, anfetaminas \\ Difenilhidantoína, metoclopramida, ciclosporina
}

IECA: inhibidores de la enzima convertidora de angiotensina; HCG: gonadotropina coriónica humana; GH: hormona de crecimiento.

TABLA 2. Resultados analíticos del paciente presentado

\begin{tabular}{lcc}
\hline Parámetro & Valor & Rango \\
\hline Prolactina & $25,52 \mu \mathrm{g} / \mathrm{L}$ & $(2,10-17,7)$ \\
LH & $<0,0 \mathrm{U} / \mathrm{L}$ & $(0,0-6,0)$ \\
FSH & $1,9 \mathrm{mU} / \mathrm{ml}$ & $(1,4-18,1)$ \\
Estradiol & $1,7 \mathrm{pg} / \mathrm{ml}$ & $(0-39)$ \\
Progesterona & $0,45 \mu \mathrm{g} / \mathrm{L}$ & $(0,26-1,22)$ \\
Testosterona & $0,08 \mathrm{ng} / \mathrm{ml}$ & $(2,41-8,27)$ \\
DHEA-S & $32,97 \mu \mathrm{g} / \mathrm{ml}$ & $(34,50-568,90)$ \\
17-OH progesterona & $1,57 \mathrm{ng} / \mathrm{ml}$ & $(0,20-0,90)$ \\
Androstendiona & $0,36 \mathrm{ng} / \mathrm{ml}$ & $(0,15-2,95)$ \\
$\beta$-hCG & $<2 \mathrm{mUI} / \mathrm{ml}$ & $(2,00-10,00)$ \\
Tirotropina & $4,29 \mathrm{mU} / \mathrm{L}$ & $(0,35-5,50)$ \\
Aspartato aminotransferasa & $29 \mathrm{U} / \mathrm{L}$ & $(13-40)$ \\
Alanina aminotransferasa & $20 \mathrm{U} / \mathrm{L}$ & $(9-40)$ \\
Urea & $50 \mathrm{mg} / \mathrm{dl}$ & $(15-50)$ \\
Creatinina & $0,49 \mathrm{mg} / \mathrm{dl}$ & $(0,50-1,30)$ \\
\hline
\end{tabular}

Se destacan como hallazgos patológicos la elevación de los niveles de prolactina y 17-OH progesterona. LH: hormona luteinizante; FSH: hormona foliculoestimulante; DHEA-S: dehidroepiandrosterona sulfato; 17-OH progesterona: 17-hidroxiprogesterona; $\beta$ - $h$ CG: fracción beta gonadotropina coriónica humana. 
proceso benigno y autolimitado, ${ }^{1}$ es un motivo de preocupación en la consulta, en muchas ocasiones, acompañada de un componente emocional, ansiedad o incluso temor. ${ }^{2}$

Con un pico de incidencia entre los 12 y los 13 años, muy distante de la edad de nuestro paciente de 6 , suele presentarse, por lo general, de forma bilateral y asintomática, concomitante con un estadio de Tanner II-III, 6-12 meses después del inicio clínico puberal, característica también ausente en nuestro caso, que presenta un estadio de Tanner I. ${ }^{2}$ La prevalencia de la ginecomastia en la edad puberal varía entre el $4 \%$ y el $69 \%, 8$ en función de variables dependientes del observador. Sin embargo, se cree que estos datos están infravalorados. Por su parte, la ginecomastia neonatal se presenta hasta en un $70 \% .{ }^{8}$ Fuera de estos períodos, su presencia es muy poco frecuente $(5 \%),{ }^{8}$ máxime cuando, como en nuestro paciente, aparece de forma unilateral. ${ }^{6}$ Las causas más frecuentes de ginecomastia en la edad prepuberal son la administración de compuestos estrogénicos, tumores testiculares secretores de hormonas esteroideas o hCG, síndrome de exceso de aromatasa e hiperandrogenismo familiar, síndromes como Klinefelter, Carney o PeutzJeghers, pubertad precoz o tumores mamarios benignos, entre otros. ${ }^{4}$

Para llegar al diagnóstico, es primordial una exhaustiva anamnesis, clave en el caso presentado, e indagar en la forma de inicio, la evolución, los síntomas asociados, locales o sistémicos, la exposición a fármacos o sustancias (tópicos o sistémicos, por parte del paciente o por convivientes), los antecedentes familiares de ginecomastia y personales de patología de base que afecte la síntesis y el metabolismo hormonal masculino. ${ }^{7,9,10}$

La adecuada exploración física conlleva palpar la glándula con el paciente en decúbito para diferenciar la ginecomastia real de la pseudoginecomastia. En caso de tumor irregular, de consistencia dura, adherido a los planos profundos, con afectación de la piel y/o los ganglios locorregionales, se debe descartar la causa oncológica. Además, se debe reseñar la antropometría y el estadio puberal.

En cuanto a las pruebas complementarias, no se deben realizar de rutina, sino solo en aquellos casos de gran tamaño, crecimiento rápido, evolución tórpida, períodos no fisiológicos, con signos de organicidad o patología sistémica, o si persisten dudas tras una detallada historia clínica. ${ }^{9}$ De ser preciso, se recomienda pedir una analítica sanguínea ${ }^{11}$ que recoja los datos solicitados en nuestro caso, así como el volumen de sedimentación globular (VSG) o proteína $C$ reactiva (PCR) para descartar un proceso inflamatorio, cariotipo si existe hipogonadismo, o cortisol libre urinario y hormona adrenocorticotropa (adrenocorticotropic hormone; ACTH, por sus siglas en inglés) si existe obesidad. Como se ha comentado, en nuestro caso, solo se destaca una hiperprolactinemia, secundaria a la estimulación continua de la glándula, que justifica el aumento de tamaño mamario, que puede, en ocasiones, llegar a la producción láctea. En casos graves y, sobre todo, en la edad adulta, esta está implicada en la patogénesis por inducir hipogonadismo secundario y por la presencia de receptores de esta en el tejido mamario masculino, ${ }^{12}$ que se cree que son corregulados y coexpresados con hormona de crecimiento (growth hormone; $\mathrm{GH}$, por sus siglas en inglés) y progesterona. A su vez, la activación de receptores de progesterona reduce la expresión de los de andrógenos, y se dificulta la inhibición del crecimiento mamario por parte de estos últimos. ${ }^{12}$

Debe realizarse una ecografía mamaria o testicular si se sospecha neoplasia en el área y tomografía computarizada (TC) si existe la sospecha de lesiones suprarrenales $\mathrm{u}$ otra localización extragonadal. Las pruebas de imagen de silla turca ${ }^{12}$ se plantean en caso de alteración de las hormonas hipofisarias para descartar procesos orgánicos en dicho nivel.

En nuestro paciente, una vez descartadas otras causas de hiperprolactinemia, podría plantearse la resonancia magnética (RM) o la TC (mucho menos efectiva). Sin embargo, este aportaba una radiografía lateral de cavum, prueba que solo podría mostrar hallazgos en casos de macroadenomas de gran tamaño causantes de expansión y erosión selar, por lo que no sería útil para un diagnóstico inicial, ya que, además de su radiación, presenta una muy alta tasa de falsos negativos. Además, en nuestro caso, en el que se sospechaba que la causa de la hiperprolactinemia y la ginecomastia secundaria era periférica, no habría sido necesaria, inicialmente, una prueba de imagen a nivel hipofisario.

En cuanto al tratamiento de la ginecomastia, consiste en corregir la causa subyacente, retirando el agente inductor o corrigiendo las alteraciones endocrinológicas responsables. Se debe tener en cuenta que la administración de testosterona en caso de hipogonadismo primario o secundario 
podría ser incluso contraproducente y aumentar por aromatización de los andrógenos. ${ }^{6}$

Si bien el tratamiento debe individualizarse, ${ }^{11}$ dado que la mayoría se resuelve espontáneamente, se reserva el manejo médico-quirúrgico para pacientes con causa orgánica o idiopática de peor pronóstico, como aquellos con antecedentes familiares positivos, macroginecomastia $(>4 \mathrm{~cm})$, síntomas invalidantes y larga duración. Pasados los 12 meses de evolución, la mama se vuelve fibrótica, con menor respuesta al tratamiento médico. ${ }^{8}$ Este, de uso limitado y muy discutido, se basa en antiandrógenos, bloqueantes estrogénicos o inhibidores de la aromatasa. ${ }^{8,9}$ Está contraindicado el tratamiento con testosterona, pues no ha demostrado mayor tasa de respuesta que en pacientes no tratados y conlleva riesgo de ser aromatizado a estradiol, lo que empeora el cuadro. ${ }^{1}$

Sin embargo, en casos crónicos o graves, con mala respuesta al tratamiento farmacológico, se recurre a la cirugía, con aceptables resultados estéticos y funcionales.

Por su parte, el manejo de la ginecomastia mecánica, como en nuestro caso, es, generalmente, conservador, ${ }^{11}$ salvo que presente infección subyacente, necrosis tisular o gran hematoma, que requeriría el desbridamiento quirúrgico.

\section{CONCLUSIONES}

- Es fundamental una buena historia clínica para detectar la etiología del cuadro.

- En caso de ginecomastia unilateral, con signos de gravedad o en edad no lactante ni púber, es necesario hacer el despistaje tumoral, a no ser que se detecte una causa clara.
- Una causa poco frecuente de ginecomastia es la hiperprolactinemia por estimulación continua de la glándula, la cual induce su crecimiento e incluso la secreción láctea.

- El tratamiento de la ginecomastia mecánica implica la eliminación del factor desencadenante.

\section{REFERENCIAS}

1. Braunstein GD. Gynecomastia. N Engl J Med. 1993; 328(7):490-5.

2. Comité Nacional de Endocrinología. Enfoque práctico del manejo de la ginecomastia. Seis preguntas que debe responderse el pediatra ante un paciente con ginecomastia. Arch Argent Pediatr. 2011; 109(4):365-8.

3. Sansone A, Romanelli F, Sansone M, et al. Gynecomastia and hormones. Endocrine. 2017; 55(1):37-44.

4. Demirbilek H, Bacak G, Baran RT, et al. Prepubertal Unilateral Gynecomastia: Report of 2 Cases. J Clin Res Pediatr Endocrinol. 2014; 6(4):250-3.

5. Kang M, Lee C, Hwang IT, et al. Prepubertal unilateral gynecomastia in the absence of endocrine abnormalities. Ann Pediatr Endocrinol Metab. 2014; 19(3):159-63.

6. Ferraro GA, De Francesco F, Romano T, et al. Clinical and surgical management of unilateral prepubertal gynecomastia. Int J Surg Case Rep. 2014; 5(12):1158-61.

7. Pellegrin MC, Naviglio S, Cattaruzzi E, et al. A Teenager with Sudden Unilateral Breast Enlargement. J Pediatr. 2017; 182:394.

8. Barasoain Millán A. Ginecomastia. An Pediatr Contin. 2012; 10(4):183-91.

9. Ma NS, Geffner ME. Gynecomastia in prepubertal and pubertal men. Curr Opin Pediatr. 2008; 20(4):465-70.

10. Erol S, Orhan E, Sevin A, et al. Trauma: a new pseudogynecomastia cause. Aesthetic Plast Surg. 2010; 34(3):404-5.

11. Warren R, Degnim AC. Uncommon benign breast abnormalities in adolescents. Semin Plast Surg. 2013; 27(1):26-8.

12. Fideleff $H$, Suárez M, Llano M, et al. Hiperprolactinemia en niñosy adolescentes, conceptos e interrogantes actuales. Rev Argent Endocrinol Metab. 2007; 44(2):94-106. 